\title{
Estimación del almacenamiento de carbono en la biomasa de macrófitas en la Bahía interior de Puno, lago Titicaca
}

\section{Estimation of carbon storage in macrophyte biomass in the interior Bay of Puno, Titicaca lake}

\section{Ingrid Maldonado- Jimenez ${ }^{1 *}$ (D) \\ Martha Elizabeth Aparicio- Saavedra' ${ }^{2}[$}

${ }^{1}$ Escuela de posgrado de la Universidad Nacional del Altiplano de Puno. Av Floral 1153, CP. 21001. Puno, Perú.

2Programa Académico de Ecología - Facultad de Ciencias Biológicas de la Universidad Nacional del Altiplano -

Puno. Av. Floral 1153 - ciudad universitaria CP. 21001. Puno, Perú.

*Autor de correspondencia: Imaldonado@unap.edu.pe

\section{Artículo científico}

Recibido: 09 de febrero 2021 Aceptado: 08 de junio 2021

Como citar: MaldonadoJimenez I, Aparicio-Saavedra ME (2021) Estimación del almacenamiento de carbono en la biomasa de macrófitas en la Bahía interior de Puno, lago Titicaca. Ecosistemas y Recursos Agropecuarios 8(2): e2848. DOI: 10.19136/era.a8n2.2848
RESUMEN. El estudio se realizó en la Bahía interior de Puno del lago Titicaca, ubicada a orillas de la ciudad de Puno, cuyas actividades como la emisión de $\mathrm{CO}_{2}$ a la atmósfera afectan esta zona del lago. El objetivo fue estimar la capacidad de almacenamiento de carbono en las macrófitas, Schoenoplectus tatora, Potamogeton strictus, Myriophyllum elatinoides y Elodea potamogeton. Se realizaron muestreos en cinco zonas de la Bahía interior de Puno, con dos repeticiones en cada punto, la toma de muestra se realizó en los meses de: agosto, setiembre, octubre y noviembre. Para estimar el contenido de carbono en cada macrófita, se determinó la cantidad de materia orgánica en porcentaje por el método de calcinación, y se multiplicó con el factor de conversión de 1.724. Las variables consideradas en el estudio fueron: contenido de biomasa, carbono, $\mathrm{CO}_{2}, \mathrm{pH}$, temperatura, profundidad y transparencia; los porcentajes de conversión de carbono hallados fueron del $55 \%$ para S. tatora, $48 \%$ para E. potamogeton, $47.5 \%$ para M. elatinoides y $47.4 \%$ para $P$. strictus. Los resultados muestran que: $S$. tatora es la especie que mayor cantidad de carbono ha capturado con un máximo de $1276 \mathrm{~g} \cdot \mathrm{m}^{2}$, M. elatinoides con un valor máximo de 298.8 g.m $\mathrm{m}^{2}$, E. potamogeton con $313.7 \mathrm{~g} . \mathrm{m}^{2}$ y P. strictus con $84.5 \mathrm{~g} \cdot \mathrm{m}^{2}$; influyendo en mayor proporción la profundidad y transparencia. Por lo que $S$. tatora fue la especie que mayor cantidad de carbono almacenó en su biomasa y por metro cuadrado en el área estudiada.

Palabras clave: Almacenamiento, biomasa, carbono, $\mathrm{CO}_{2}$, macrófitas.

ABSTRACT. The study was conducted in the Puno Inland Bay of Lake Titicaca, located on the shores of the city of Puno, whose activities such as the emission of $\mathrm{CO}_{2}$ into the atmosphere affect this area of the lake. The objective was to estimate the carbon storage capacity of macrophytes, Schoenoplectus tatora, Potamogeton strictus, Myriophyllum elatinoides and Elodea potamogeton. Sampling was carried out in five areas of the inner Bay of Puno, with two replicates at each point, sampling was done in the months of August, September, October and November. To estimate the carbon content in each macrophyte, the amount of organic matter in percentage was determined by the calcination method, and multiplied with the conversion factor of 1.724 . The variables considered in the study were: biomass content, carbon, $\mathrm{CO}_{2}, \mathrm{pH}$, temperature, depth and transparency; the carbon conversion percentages found were $55 \%$ for S. tatora, $48 \%$ for $E$. potamogeton, $47.5 \%$ for $M$. elatinoides and $47.4 \%$ for $P$. strictus. The results show that: $S$. tatora is the species that captured the greatest amount of carbon with a maximum of $1276 \mathrm{~g} . \mathrm{m}^{2}, M$. elatinoides with a maximum value of $298.8 \mathrm{~g} \cdot \mathrm{m}^{2}, E$. potamogeton with $313.7 \mathrm{~g} \cdot \mathrm{m}^{2}$ and $P$. strictus with $84.5 \mathrm{~g} \cdot \mathrm{m}^{2}$; influencing in greater proportion the depth and transparency. Therefore, $S$. tatora was the species that stored the greatest amount of carbon in its biomass and per square meter in the area studied.

Key words: Biomass, carbon, $\mathrm{CO}_{2}$, macrophytes, storage. 


\section{INTRODUCCIÓN}

En los últimos años se han incrementado las emisiones de $\mathrm{CO}_{2}$ al ambiente; al respecto, la Organización Meteorológica Mundial (OMM) reportó que entre los años 2019-2020, los niveles de $\mathrm{CO}_{2}$ en la atmósfera alcanzaron niveles récord de $410 \mathrm{ppm}$, que equivale a un $148 \%$ más, comparados con los niveles preindustriales (OMM 2021). Este aumento se debe principalmente a diversas actividades humanas, como el uso de combustible para el sistema de transporte vehicular, deforestación e incendios (Montero et al. 2005), además de grandes cantidades de dióxido de carbono, que se liberan por la degradación de los humedales, lo que contribuye al aumento de la temperatura mundial (PalominoContreras y Cabrera-Carranza 2007), dado que el $\mathrm{CO}_{2}$ es un gas de efecto invernadero que contribuye con el calentamiento global (Díaz-Franco et al. 2016).

En contraparte los bosques tienen gran capacidad de almacenamiento de carbono. Al respecto, un estudio realizado en bosques naturales de Honduras, reportan un secuestro anual de $\mathrm{CO}_{2}$ de $105989.86 \mathrm{t}$ (Alberto y Elvir 2008); pero estos, no son los únicos ecosistemas que proveen ese servicio. También, los humedales juegan un rol importante por su alta capacidad de almacenamiento de carbono, por medio de las macrófitas y el fitoplancton (Medrano et al. 2012, Lolu et al. 2019); así como, los manglares que son muy eficientes en este proceso a largo tiempo, como lo indica Rovai et al. (2021). En los humedales se reportan diversas macrófitas, por ejemplo en la Bahía de Cohana del lago Titicaca, se halló hasta 58 especies (Fontúrbel et al. 2006), las cuales almacenan carbono y contribuyen de forma significativa en la retención de carbono, al mismo tiempo que brinda un servicio ambiental (Palomino-Contreras y Cabrera-Carranza 2007). Aunque dependiendo de la especie de macrófitas, se sabe que algunas contribuyen en mayor porcentaje que otras, como es el caso de las especies emergentes (Maqbool y Khan 2013) teniendo éstas mejores resultados en monocultivos y en zonas controladas (Means et al. 2016).

Los humedales brindan diversos servicios eco- sistémicos, como el almacenamiento de carbono (Cejudo y Herrera-Caamal 2019, Lin et al. 2019, Lund y Drake 2020), por lo que algunos autores, las consideran como uno de los principales sumideros de carbono del planeta ( Mitsch et al. 2013, DvořákováBřezinová y Vymazal 2018). Lo que se debe principalmente a la descomposición incompleta de la biomasa vegetal en los sedimentos, la presencia de macrófitas y fitoplancton, los que contribuyen en el almacenamiento de carbono (Calva Benítez y Torres Alvarado 2011, Dvořáková Březinová y Vymazal 2018, Mitsch et al. 2013). Dentro de los cuales, las macrófitas emergentes son las que fijan la mayor cantidad de carbono que las sumergidas (Maqbool y Khan 2013), dado que éstas últimas están condicionadas al paso de la luz y al carbono disuelto en el agua (Liu et al. 2020, Madsen et al. 1996). Pero los estudios realizados en humedales y macrófitas fijadoras de carbono en muchos lugares son escasos, por lo que es necesario realizar estudios para entender mejor los beneficios que brindan estos ecosistemas (Lolu et al. 2019).

En el lago Titicaca se encuentra una gran cantidad de macrófitas, esencialmente totorales y llachales, cuya presencia permite mitigar los impactos que provienen de las actividades antropogénicas (Goyzueta et al. 2009), como la emisión de gases por el sistema vehicular y vertimiento de aguas residuales, que afectan el desarrollo de las especies predominantes de la zona. La biomasa de macrófitas es abundante, principalmente en la zona de orilla (Dejoux y lltis 1991), lo que contribuye con el proceso de captura de $\mathrm{CO}_{2}$; dado que, cierto porcentaje de la biomasa es carbono (PELT y TDPS 2000, PELT y ADESU 2001), aproximadamente el 50\% (Dávalos et al. 2008); lo que permite entender, parte de los beneficios ambientales que proporcionan estos ecosistemas. Por lo anterior, el objetivo del estudio fue estimar la concentración de carbono almacenado en la biomasa de cuatro especies de macrófitas predominantes en la Bahía interior de Puno y la variación de almacenamiento de carbono entre ellas, para conocer el aporte de estas macrófitas en la mitigación de $\mathrm{CO}_{2}$ en el ambiente. 


\section{MATERIALES Y MÉTODOS}

\section{Área de estudio}

La zona de estudio se encuentra en el lago Titicaca, conocida como; Bahía interior de Puno. Tiene un área de $17.5 \mathrm{~km}^{2}$ (Ocola-Salazar y LaquiVilca 2017), profundidad máxima de $7.19 \mathrm{~m}$ (Loza del carpio et al. 2016); temperatura entre 12 y $13^{\circ} \mathrm{C}$ y precipitación anual de 700 a $1000 \mathrm{~mm}$. Con diversidad de flora y fauna, de las cuales para el estudio se tomó en cuenta las cuatro especies de macrófitas más dominantes del área, que son, Schoenoplectus tatora, Elodea potamogeton, Myriophyllum elatinoides y Potamogeton strictus. La ciudad de Puno está asentada a orillas de la Bahía, con actividades antrópicas, como el vertimiento de aguas residuales y emisión de gases provenientes del conjunto de los automóviles que transitan por la ciudad y de la quema ilegal de totorales. La zona fue elegida de acuerdo a los impactos antrópicos que existen, los cuales influencian el almacenamiento de carbono, principalmente el vertimiento de aguas residuales provenientes de la ciudad de Puno.

\section{Diseño de muestreo}

Se seleccionaron cinco puntos de muestreo dentro del área de la Bahía interior de Puno, de acuerdo a la influencia de actividades antrópicas presentes en la zona (Tabla 1). El muestreo se realizó durante los meses de agosto, setiembre, octubre y noviembre del 2011, se colectaron muestras por duplicado, de Schoenoplectus tatora, Elodea potamogeton, Myriophyllum elatinoides y Potamogeton strictus, de las cuales se tomaron muestras de tallos y hojas mediante muestreo directo en cuadrantes de 1 $\mathrm{m}^{2}$, para luego extraer la parte sumergida, así como la parte emergente comprendidos dentro del área del cuadrante (sin rizomas); para luego guardar las muestras en depósitos de plástico y luego trasladarlas al laboratorio para su análisis. La temperatura se registró con un termómetro digital marca thermometer herwowe, el $\mathrm{pH}$ con un potenciómetro marca Hanna, la transparencia del agua fue medida con un Disco Secchi, cuya medida es el promedio de la profundidad a la que el ojo humano deja de ver el disco metálico ( $20 \mathrm{~cm}$ de diámetro) al ser introducido en el agua, y la profundidad en la que aparece de nuevo al ojo humano (Lee et al. 2015), las coordenadas se registraron con un GPS marca Garmin etrex 30 y la profundidad se midió con una barra de madera milimétrica, dado que las mayores áreas de esta zona están ocupadas por profundidades menores a $1 \mathrm{~m}$, como máximo $4 \mathrm{~m}$ (Loza-Del-Carpio et al. 2016). Todos estos parámetros se midieron en la zona de muestra de la planta, en la columna de agua, antes de tomar las muestras de macrófitas, para no alterar las mediciones.

\section{Análisis en laboratorio}

En el laboratorio se determinó el peso de la biomasa húmeda (Drexler et al. 2021), después se pusieron las muestras en una estufa de convección natural a $75^{\circ} \mathrm{C}$, hasta obtener peso constante para determinar la biomasa seca. Luego se dividió la muestra en cuatro submuestras por especie, las cuales se pulverizaron en un mortero, se tomó su peso inicial; para posteriormente llevar las submuestras a una mufla para calcinarlas a $400{ }^{\circ} \mathrm{C}$ y determinar el peso de cenizas para calcular la materia orgánica de cada especie, que se calculó como la diferencia entre el peso inicial y final de la muestra dividido entre el peso de la muestra inicial. Determinada la materia orgánica en la biomasa seca, se determinó la cantidad de carbono almacenado por especie en $\mathrm{g} \mathrm{m}^{2}$, con la siguiente fórmula

$$
\% C=\% M . O . * 1.724 .
$$

Dónde: \% C = porcentaje de carbono por especie, $\%$ M.O.= porcentaje de materia orgánica y $1.724=$ constante (Palomino y Cabrera 2007).

Con la cantidad de carbono almacenado, se determina la cantidad de $\mathrm{CO}_{2}$ capturado en unidades de gramos $\left(\mathrm{g} \mathrm{m}^{2}\right)$ con la fórmula:

$$
\mathrm{CO}_{2}=\mathrm{KrxC} .
$$

Donde: $\mathrm{CO}_{2}=$ Dióxido de carbono, $\mathrm{C}=$ Carbono, $\mathrm{Kr}$ $=3.67$, factor de conversión a $\mathrm{CO}_{2}$, resultante del cociente de los pesos moleculares del dióxido de carbono 44 y del carbono 12. Dado que una molécula 
Tabla 1. Descripción de los puntos de muestreo en la Bahía interior de Puno del lago Titicaca.

\begin{tabular}{|c|c|c|c|c|}
\hline \multirow[t]{2}{*}{$\begin{array}{l}\text { Puntos de } \\
\text { muestreo }\end{array}$} & \multicolumn{2}{|c|}{$\begin{array}{l}\text { Coordenadas } \\
\text { geográficas }\end{array}$} & \multirow{2}{*}{$\begin{array}{l}\text { Zonas } \\
\text { de } \\
\text { muestreo }\end{array}$} & \multirow[t]{2}{*}{ Descripción } \\
\hline & $\mathrm{X}$ & $\mathrm{Y}$ & & \\
\hline P1 & 8249802 & 393464 & Isla Esteves & $\begin{array}{l}\text { Con tránsito de lanchas motorizadas. Además, por esta zona existen hoteles turísticos que } \\
\text { vierten sus aguas servidas directamente al lago sin tratamiento alguno, con un } \mathrm{pH} \text { promedio } \\
\text { de } 8.08 \text {, transparencia de } 0.54 \mathrm{~m} \text { profundidad } 0.87 \mathrm{~m} \text {. }\end{array}$ \\
\hline P2 & 8248861 & 394276 & Isla Joroba & $\begin{array}{l}\text { En esta área existe gran cantidad de totorales, está cerca de la ruta de transito de las em- } \\
\text { barcaciones que zarpan desde el muelle de Puno con dirección a las islas flotantes de los } \\
\text { Uros; con un pH promedio de } 8.09 \text {, transparencia } 0.71 \mathrm{~m} \text {, profundidad } 1.39 \mathrm{~m} \text {. }\end{array}$ \\
\hline P3 & 8247028 & 395035 & Cerca de Chimu & $\begin{array}{l}\text { Esta zona se halla cerca de la laguna de oxidación de la ciudad de Puno. No es la zona con } \\
\text { la transparencia mas alta, hay otros puntos con > transparencia. Tiene un pH promedio de } \\
7.96 \text {, transparencia } 0.58 \mathrm{~m} \text {, profundidad } 1.47 \mathrm{~m} \text {. }\end{array}$ \\
\hline $\mathrm{P} 4$ & 8247364 & 393083 & $\begin{array}{l}\text { Cercanías de la } \\
\text { Isla espinar }\end{array}$ & $\begin{array}{l}\text { Cerca de este punto está el efluente de aguas residuales de la ciudad de Puno; las macrófi- } \\
\text { tas que existen por esa zona son los totorales. Con un } \mathrm{pH} \text { promedio de } 7.97 \text {, transparencia } \\
0.42 \mathrm{~m} \text {, profundidad } 1.19 \mathrm{~m} \text {. }\end{array}$ \\
\hline P5 & 8249257 & 396947 & $\begin{array}{l}\text { Malecón Eco- } \\
\text { turístico }\end{array}$ & $\begin{array}{l}\text { Es una zona recreativa, en el cual se emplea cayacs. También en esta zona se realizó un } \\
\text { tratamiento con microorganismos eficientes, cuyos resultados hasta la fecha son positivos, } \\
\text { pues a la observación directa el agua se muestra transparente, así como la presencia de } \\
\text { macrófitas e incluso especies de peces. Con un pH promedio de } 7.78 \text {, transparencia } 0.27 \\
\mathrm{~m} \text {, profundidad } 0.27 \mathrm{~m} \text {. }\end{array}$ \\
\hline
\end{tabular}

de Carbono pesa $12 \mathrm{~g} \cdot \mathrm{mol}^{-1}$ y una molécula de $\mathrm{CO}_{2}$ pesa $44 \mathrm{~g} \cdot \mathrm{mol}^{-1}$, luego $44 / 12=3.67$, una tonelada de carbono fijada en un árbol equivale a fijar 3.67 toneladas de $\mathrm{CO}_{2}$.

\section{Análisis estadístico}

Se usaron las pruebas de KolmogorovSmirnov y el Test de Levene, para comprobar la distribución normal y la homogeneidad de varianzas, las cuales indicaron que los datos no cumplían con los criterios para aplicar una prueba paramétrica, por lo que se aplicó el análisis de Kruskal Wallis con un nivel de confianza del $95 \%$, para luego realizar la prueba de contraste de Kruskalmc (Prueba de comparación Múltiple después del Kruskal-Wallis) con un nivel de significancia de $P<0.05$. También se realizó una correlación de Pearson para relacionar el carbono almacenado en la biomasa de macrófitas y variables ambientales, como profundidad y trasparencia. Los análisis se realizaron en el software libre $R$ versión 4.0.3.

\section{RESULTADOS}

\section{Almacenamiento de carbono en biomasa seca de macrófitas}

Para el carbono almacenado en las macrófitas $S$. tatora es la especie con mayor porcentaje de car- bono en su biomasa seca con $55 \%$, seguida de $E$. Potamogeton con $48 \%$, M. elatinoides con $47.5 \%$, y P. strictus con un $47.4 \%$ (Figura 1).

\section{Almacenamiento de carbono en macrófitas}

En relación al carbono almacenado por $\mathrm{m}^{2}$, los resultados indican que $S$. tatora tiene la mayor cantidad y variación, ya que su valor máximo fue de 1 276 g.m ${ }^{2}$ y su valor mínimo de 44 g.m², seguida de E. potamogeton con máximo y mínimo de 313.7 y 41.28 g. $\mathrm{m}^{2}$, M. elatinoides con valor máximo y mínimo de 96.9 y 8.07 g.m², y $P$. strictus con valor máximo y mínimo de 84.5 y $7.7 \mathrm{~g} . \mathrm{m}^{2}$, respectivamente (Figura 2). Estos resultados indican que el contenido de carbono es significativamente superior en $S$. tatora con media de $629.4 \mathrm{~g} . \mathrm{m}^{2}$, seguida de E. Potamogeton con 109.8 g.m ${ }^{2}, M$. elatinoides con 45.11 g.m $\mathrm{m}^{2}$, y $P$. strictus con $34.5 \mathrm{~g} . \mathrm{m}^{2}$. La prueba de Kruskal Wallis; mostró diferencias significativas (Kruskal Wallis $=1.58^{e-12}, \mathrm{P}<0.05$ ); lo que indica que por lo menos una especie es diferente al resto con respecto al contenido de carbono almacenado en su biomasa seca. Para verificar las diferencias y/o similitudes, se realizó la prueba de rango múltiple de Kruskalcm, la cual detectó significancia entre $S$. tatora con las otras especies $(p<0.05)$, lo cual se visualiza en la Tabla 2; donde se observa que $S$. tatora es la que presenta el mayor almacenamiento de car- 


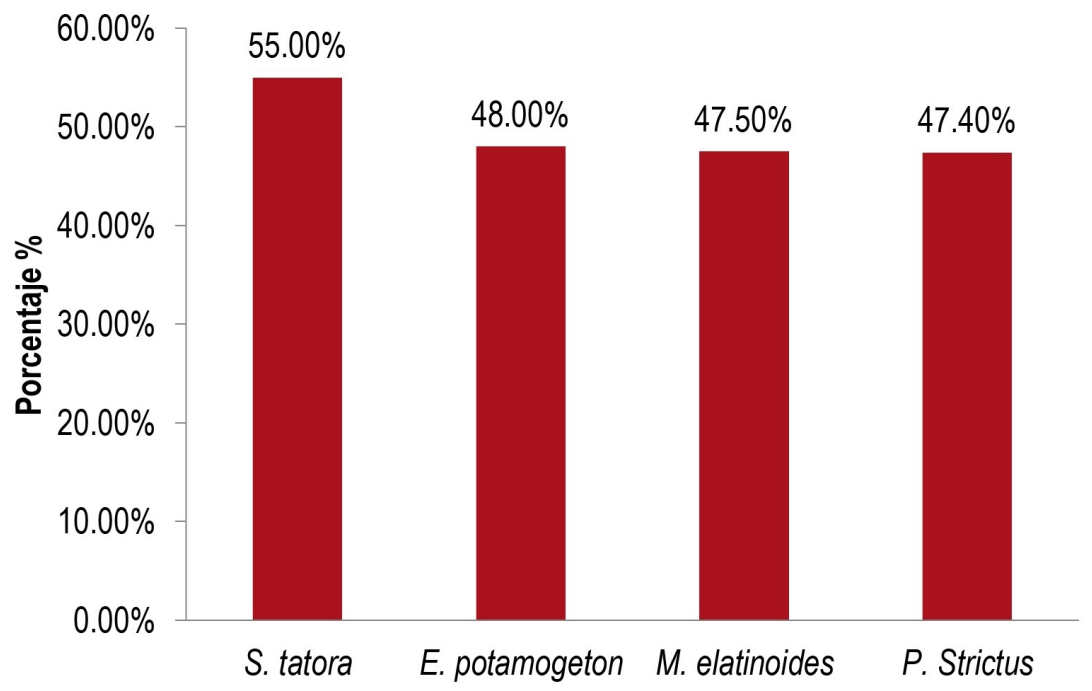

Figura 1. Porcentaje de carbono en la biomasa seca de cuatro especies de macrófitas.

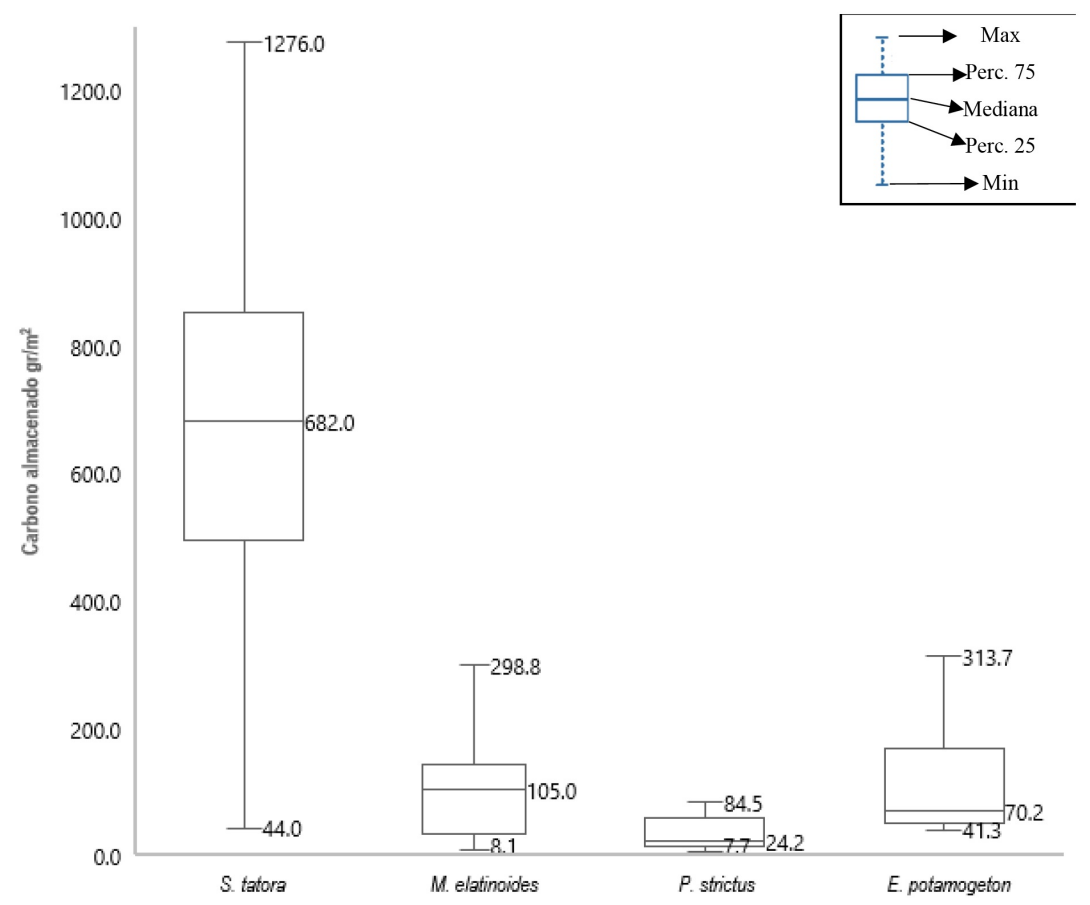

Figura 2. Comparación de almacenamiento de carbono en macrófitas predominantes en la Bahía interior de Puno del lago Titicaca.

bono; debido a su concentración en la biomasa.

Para el $\mathrm{CO}_{2}$ fijado, los resultados muestran una tendencia similar a la del carbono almacenado, observándose que $S$. tatora es la especie que mayor cantidad de $\mathrm{CO}_{2}$ captura del ambiente, con media de 2309.97 g.m², seguida de E. potamogeton con 402.98 g.m ${ }^{2}, M$. elatinoides con 375.8 g. $\mathrm{m}^{2}$ y $P$. strictus con media de $126.70 \mathrm{~g} \cdot \mathrm{m}^{2}$. Esta variación puede deberse a que la fijación de $\mathrm{CO}_{2}$ se da mejor en plantas acuáticas de tallos emergentes que las 
Tabla 2. Prueba de comparación múltiple Kruskalmc entre el carbono almacenado por macrófitas.

\begin{tabular}{|c|c|c|c|}
\hline Comparaciones & Dif. Obs & Dif.critica & Diferencia \\
\hline Elodea-Miriophyllum & 14.025 & 27.33282 & False \\
\hline Elodea-Potamogeton & 17.275 & 27.33282 & False \\
\hline Elodea-Schoenoplectus & 87.000 & 27.33282 & True* \\
\hline Miriophyllum-Potamogeton & 3.250 & 27.33282 & False \\
\hline Miriophyllum-Schoenoplectus & 72.975 & 27.33282 & True $^{*}$ \\
\hline Potamogeton-Schoenoplectus & 69.725 & 27.33282 & True * \\
\hline
\end{tabular}

sumergidas, ya que las plantas sumergidas están supeditados a factores adversos que pudieran perjudicar su normal desarrollo, como la transparencia, $\mathrm{pH}$, temperatura, nutrientes, contaminación, presión atmosférica, entre otras.

\section{Carbono almacenado en los cinco puntos de es- tudio}

La especie que mayor cantidad de carbono almacenó en los cinco puntos de muestreo fue $S$. tatora, principalmente en el punto tres se tuvo una media de $825 \mathrm{~g} \cdot \mathrm{m}^{2}$, y la menor cantidad en el punto cinco con valor medio de116.6 g.m ${ }^{2}$ (Figura 3). Esto puede deberse a que el punto tres está cerca de la laguna de oxidación por lo que hay mayor cantidad de nutrientes necesarios para su desarrollo; además de que tienen profundidades apropiadas para el desarrollo de esta especie, a pesar de la época de estiaje la variación del nivel del agua no es significativa. Pero en el punto cinco, la variación de la profundidad si fue notoria, lo que pudo influir en sus bajos niveles de captación de carbono.

Con respecto a $P$ estrictus, se aprecia que, en el segundo punto de muestreo tuvo la mayor cantidad de carbono con media de 32.9 g. $\mathrm{m}^{2}$, mientras que en las zonas tres y cuatro no se registra esta especie; lo cual pudiera estar influenciado por el ingreso de aguas residuales a esas zonas. Mientras que, los puntos uno y dos están distantes de este foco contaminante; por lo que, al tener menor influencia, hay mayor transparencia en la columna de agua de 54 $\mathrm{cm}$ en el punto uno y $70 \mathrm{~cm}$ en el punto dos, lo cual repercute en el mayor paso de la luz; lo que favorece su desarrollo. En tanto, que en las zonas de muestro tres y cuatro la profundidad fue mayor y la transparencia menor por la contaminación de aguas residuales. En lo que respecta a $M$. elatinoides, solo se encontró en el punto uno y dos, los cuales tienen aguas transparentes, con mayor oxigenación y están alejadas de la laguna de oxidación, lo cual explicaría su presencia, más no en los otros puntos de estudio. Mientras que E. potamogeton, solo se encontró en el punto cinco, lo que se puede deber a que no es una especie exigente en cuanto a nutrientes, pero si con respecto a la transparencia y temperatura, además de que requiere aguas superficiales. Lo que puede explicar que no se encontró en los primeros cuatro puntos de muestreo, ya que la temperatura promedio fue de $13^{\circ} \mathrm{C}$, mientras que en el malecón ecoturístico el promedio de temperatura fue de $15^{\circ} \mathrm{C}$, con poca profundidad, lo que contribuyó a su desarrollo.

Los resultados de la correlación de Pearson para la cantidad de carbono almacenado y parámetros como profundidad, $\mathrm{pH}, \mathrm{y}$ transparencia (Tabla 3), corroboran la explicación del porqué no se encuentra algunas especies en los puntos de estudio mencionado; como $S$. tatora, $M$. elatinoides y $P$. strictus que presentan correlación positiva con la profundidad de 79,81 y $80 \%$, respectivamente. Lo que indica, que a mayor profundidad mayor desarrollo de la especie. En tanto que E. potamogeton tiene una relación débil pero inversa, es decir, que a mayor profundidad menor almacenamiento de carbono. El pH no muestra influencia, mientras que la transparencia tiene leve relación positiva con las especies. En lo referente a $E$. potamogeton no se consideró en este análisis debido a que hubo $100 \%$ de transparencia en todas las mediciones realizadas. 


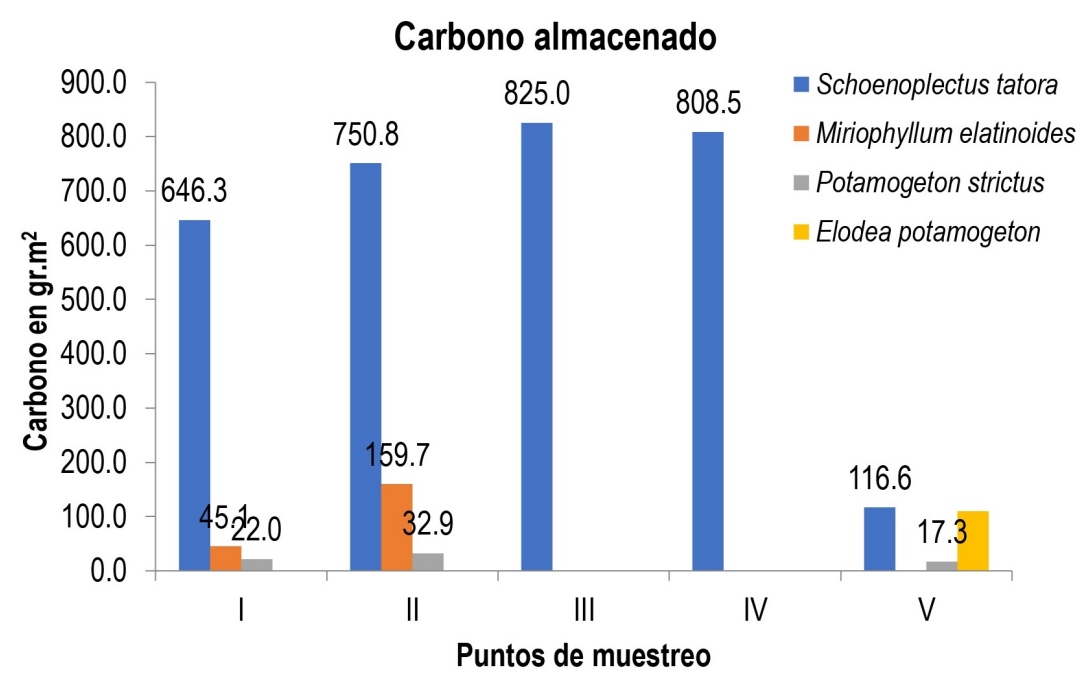

Figura 3. Almacenamiento de carbono en macrófitas, en cinco puntos de muestreo de la Bahía interior de Puno del lago Titicaca.

Tabla 3. Correlación de Pearson entre la cantidad de carbono almacenado por las macrófitas y variables ambientales.

\begin{tabular}{cccc}
\hline Especies & Profundidad & $\mathrm{pH}$ & Transparencia \\
\hline Schoenoplectus tatora & 0.79464 & 0.16059 & 0.51961 \\
$p$-valor & $9.252 \mathrm{e}-10$ & 0.3222 & 0.0005902 \\
Miriophyllum elatinoides & 0.81481 & 0.04756 & 0.47810 \\
$p$-valor & 0.0001209 & 0.8611 & 0.06105 \\
Potamogeton strictus & 0.79715 & 0.08181 & 0.19400 \\
$p$-valor & $0.117 \mathrm{e}-06$ & 0.7039 & 0.3637 \\
Elodea potamogeton & -0.38577 & 0.18596 & - \\
$p$-valor & 0.3452 & 0.6593 & - \\
\hline
\end{tabular}

\section{DISCUSIÓN}

\section{Almacenamiento de carbono en biomasa seca de macrófitas}

El porcentaje de carbono en la biomasa seca de macrófitas, no está lejos de los resultados reportados en vegetales, al respecto el Panel intergubernamental de cambio climático (IPCC) así como otros estudios, reportan que el porcentaje promedio de carbono almacenado en vegetales es el $50 \%$ de la biomasa seca (IPCC 2005, Dávalos et al. 2008, Tobías-Baeza et al. 2019). Mientras que DávalosSotelo et al. (2008), reportan que el contenido de carbono a nivel de especie puede variar del 40.27 al $46.29 \%$ e incluso puede ser mayor de $50 \%$; en tanto que Mota et al. (2011) indican que el porcentaje de carbono almacenado puede oscilar entre el 45 y $50 \%$ del peso seco de la planta. Las diferencias reportadas en este estudio se pueden deber a que las especies estudiadas tienen diferentes características anatómicas y fisiológicas, con necesidades y condiciones a las que deben de adaptarse. Por lo que, el promedio porcentual de conversión de carbono es del $50 \%$, pudiendo oscilar por debajo o por encima de este valor, como se encontró en este estudio.

\section{Almacenamiento de carbono en macrófitas en los cinco puntos de muestreo}

En relación a las macrófitas y su capacidad de almacenamiento de carbono, $S$. tatora fue la especie que mayor cantidad de carbono almacenó en su biomasa por metro cuadrado. Lo que coincide con estudios realizados en humedales, los cuales indican que Schoenoplectus californicus, especie similar a la a $S$. tatora, es la que mayor carbono almacena en su biomasa, a diferencias de otras macrófitas (PalominoContreras y Cabrera-Carranza 2007, Medrano et al. 
2012). Por ello diversos estudios consideran a esta especie, debido a su extensa área de crecimiento en el lago Titicaca. Al respecto en el área de la Reserva Nacional del Titicaca existe gran cantidad de biomasa debido a la mayor profundidad y mínimos niveles de fluctuación de agua; con profundidades entre los 2 y $4 \mathrm{~m}$, que son adecuados para el desarrollo de esta especie ( PELT y TDPS 2000, Goyzueta et al. 2009); incluso el CIDAB (2003) menciona que esta macrófita puede crecer hasta cinco metros de profundidad. En tanto que $P$. strictus, prefiere zonas de aguas transparentes, mientras que Potamogeton pectinatum crece hasta $1.5 \mathrm{~m}$ de profundidad en zonas con sustrato arenoso o algo limosos (URS-CHE 2010). También se sabe que esta especie se desarrolla mejor en zonas oligotróficas ubicadas en aguas lénticas como las de la zona de estudio (PELT y TDPS 2000, San Martín et al. 2011). En tanto que M. elatinoides necesita una gran cantidad de luz, y que algunas especies de este género pueden crecer hasta 10 metros de profundidad en condiciones apropiadas (URS-CHE 2010). Al respecto el PELT y TDPS (2000) mencionan que es una especie asociada a S. tatora, que presenta tallos frágiles, difusión limitada por el movimiento de las aguas y por la dureza del substrato. En tanto que, E. potamogeton prefiere zonas someras como en las orillas de la Bahía de Cohana, el lado boliviano del lago Titicaca (Fontúrbel et al. 2006), así como en las orillas y canales de ingreso de los pantanos de Villa-Lima (Ramirez y Cano 2011). Por lo que, las especies $P$. strictus, M. elati- noides, y E. Potamogeton son de aguas oligotróficas, transparentes, dado que desarrollan su ciclo vegetativo y comportamiento fenológico dentro del agua, por lo que es necesario el paso de la luz (Fontúrbel et al. 2006), y la contaminación de la columna de agua las afectará más que a las especies con tallos emergentes.

\section{CONCLUSIONES}

La especie $S$. tatora fue la de mayor porcentaje de conversión de carbono tuvo en la biomasa seca, mientras que $P$. strictus fue la de menor porcentaje de carbono. También $S$. tatora presento la mayor cantidad de almacenamiento de carbono y $\mathrm{CO}_{2}$ por metro cuadrado. Los factores que más influyeron en la captura de carbono fueron la profundidad y turbidez. También las actividades antrópicas repercuten en la captura de carbono de las diferentes especies, debido a que la contaminación de las aguas, se desplazan a zonas de menor influencia antrópica, principalmente en el caso de las plantas sumergidas como E. potemogenton, $M$. elatinoides y $P$. strictus. Se encontró capacidad de almacenamiento de carbono en las macrófitas estudiadas; por lo que, su distribución, función y capacidad de almacenamiento son prometedoras y significativamente sostenibles; por ello deberían ser mejor valorados por presentar un servicio ambiental.

\section{LITERATURA CITADA}

Alberto DM, Elvir JA (2008) Acumulación y fijación de carbono en biomasa aérea de Pinus oocarpa en bosques naturales en Honduras. Investigación Agraria Sistemas y Recursos Forestales 17: 67-78.

Calva Benítez LG, Torres Alvarado R (2011) Carbono orgánico y características texturales de sedimentos en áreas del pasto marino Thalassia testudinum en ecosistemas costeros del sureste del Golfo de México. Universidad y Ciencia 27: 133-144.

Cejudo E, Herrera-Caamal K G (2019) Humedales en dolina del norte de Quintana Roo, México: ecosistemas poco conocidos. Ecosistemas y Recursos Agropecuarios 6: 207-21

CIDAB (2003) Conservación de la biodiversidad en la cuenca del lago Titicaca - Desaguadero - Poopo - Salar de Coipasa (TDPS). Programa de las Naciones Unidas Para el Desarrollo. Perú-Bolivia: $67 p$.

Dávalos-Sotelo R, Morato IM, Martínez-Pinillos-Cueto E (2008) Almacenamiento de carbono. En: Manson RH, 
Hernández-OrtízV, Gallina S, Mehltreter K (ed.) Agroecosistemas cafetaleros de Veracruz biodiversidad, manejo y conservación. Instituto de Ecología, Instituto Nacional de Ecología. México. pp: 223-233.

Dejoux C, Iltis A (1991) El Lago Titicaca, síntesis del conocimiento limnológico actual. ORSTOM, HISBOL. La Paz, Bolivia. 584p.

Díaz -Franco R, Acosta-Mireles M, Carrillo-Anzures F, Buendía-Rodríguez E, Flores-Ayala E, Etchevers-Barra JD (2016) Determinación de ecuaciones alométricas para estimar biomasa y carbono en Pinus patula Schl. et Cham. Madera y Bosques 13: 25-34.

Drexler JZ, Khanna S, Lacy JR (2021) Carbon storage and sediment trapping by Egeria densa Planch., a globally invasive, freshwater macrophyte. Science of the Total Environment 755: 142602. DOI: 10.1016/j.scitotenv. 2020.142602

Dvořáková-Březinová T, Vymazal J (2018) Evaluation of macrophytes suitable for agriculture drainage treatment with respect to their carbon sequestration potential. Ecological Engineering 124: 31-37.

Fontúrbel F, Richard E, García G (2006) Diversidad de flora fanerógama y aspectos ecobiológicos de la unidad vegetal de Aygachi-Cumana- bahía Cohana, lago Titikaka (La Paz, Bolivia). Ciencia Abierta Internacional 30: 1-19.

Goyzueta G, Alfaro R, Aparicio M (2009) Totorales del lago Titicaca, importancia, conservación y gestión ambiental. Primera Edición. Editorial Meru diseño y publicidad. Puno, Perú. 325p.

IPCC (2005) Orientación sobre las buenas prácticas para uso de la tierra, cambio de uso de la tierra y silvicultura. En: Jim-Penman DK, Gytarsky M, Hiraishi, Krug T, Riitta-Pipatti F, Buendia L, Miwa K Wagner TN (Eds.) Programa del IPCC sobre inventarios nacionales de gases de efecto invernadero. Ginebra - Suiza. 628p.

Lee Z P, Shang S, Hu C, Du K, Weidemann A, Hou W, Lin J, Lin G (2015) Secchi disk depth: A new theory and mechanistic model for underwater visibility. Remote Sensing of Environment 169: 139-149.

Lin Q, Zhang K, Shen Ji, Liu E (2019) Integrating long-term dynamics of ecosystem services into restoration and management of large shallow lakes. Science of the Total Environment 671: 66-75.

Liu J, Longjuan Ch, Shuchun Y, Xue B (2020) Variations in stable carbon isotopes in different components of aquatic macrophytes from Taihu Lake, China. Ecological Indicators 118: 106721. DOI: 10.1016/j.ecolind. 2020.106721.

Lolu AJ, Amrik SA, Malkiat CS, Zafar AR (2019) Carbon Sequestration Potential of Macrophytes and Seasonal Carbon Input Assessment into the Hokersar Wetland, Kashmir. Wetlands 39: 453-472.

Loza del carpio A, Gamarra C, Condori N (2016) Caracterización morfobatimétrica y estimación de sedimentos de la Bahía interior de Puno, lago Titicaca, mediante tecnología SIG. Revista de Investigaciones Altoandinas 18: $237-248$.

Lund E, Drake D (2020) Species-specific wet-dry mass calibrations for common submersed macrophytes in the Upper Mississippi River. Aquatic botany 169: 104743. DOI: 10.1016/j.aquabot.2020.103344.

Madsen TV, Maberly SC, Bowes G (1996) Photosynthetic acclimation of submersed angiosperms to $\mathrm{CO}_{2}$ and $\mathrm{HCO}^{3-}$. Aquatic Botany 53: 15-30.

Maqbool C, Khan AB (2013) Biomass and carbon content of emergent macrophytes in Lake Manasbal, Kashmir: Implications for carbon and sequestration. International Journal of Scientific and Research Publication 3: 1-7. 
Means MM, Changwoo A, Korol AR, Williams LD (2016) Carbon storage potential by four macrophytes as affected by planting diversity in a created wetland. Journal of Environmental Management 165: 133-139.

Medrano R, Chupan L, Vila M (2012) Almacenamiento de carbono en especies predominantes de flora en el lago Chinchaycocha. Apuntes de Ciencia y Sociedad 2: 110-117.

Mitsch WJ, Bernal B, Nahlik A M, Mander Ü, Zhang L, Anderson C J, Jørgensen S E, y Brix H (2013) Wetlands, carbon, and climate change. Landscape Ecology, 28: 583-597.

Montero G, Ruiz-Peinado R, Muñoz M (2005) Producción de biomasa y fijación de $\mathrm{CO}_{2}$ por los bosques españoles. Instituto Nacional de Investigación y Tecnología Agraria y Alimentaria, Ministerio de Educación y Ciencia. Madrid, España. 275p.

Mota C, Alcaraz-López MI, Martínez-Ballesta MC, Carvajal M (2011) Investigación sobre la absorción de $\mathrm{CO}_{2}$ por los cultivos más representativos de la región de Murcia. Horticultura Global 294: 58-63.

Ocola-Salazar JJ, Laqui-Vilca WF (2017) Fuentes contaminantes en la cuenca del lago Titicaca: un aporte al conocimiento de las causas que amenazan la calidad del agua del maravilloso lago Titicaca. Autoridad Nacional del Agua. Lima, Perú. 187p.

OMM. (2021) Los indicadores empeoraron y los impactos del cambio climático se agravaron en 2020. Organización Meteorológica Mundial. Ginebra, Suiza. https://public.wmo.int/es/media/comunicados-de-prensa/losindicadores-empeoraron-y-los-impactos-del-cambio-climático-se. Fecha de consulta: 10 de mayo del 2021.

Palomino-Contreras D, Cabrera-Carranza C (2007) Estimación del servicio ambiental de captura del $\mathrm{CO}_{2}$ en la flora de los humedales de Puerto Viejo. Revista del Instituto de Investigación de la Facultad de Ingeniería Geológica, Minera, Metalurgica y Geográfica 10: 49-59.

PELT, ADESU (2001) Programa de capacitación sobre el manejo de la totora - Técnicas de Reimplante. Proyecto especial binacional del lago Titicaca. ALT. Puno, Perú. 51p.

PELT, TDPS (2000) Proyecto conservación de la biodiversidad en el Sistema TDPS evaluación de la totora en el Perú. PELT, ALT, PNUD. Puno, Perú. 78p.

Ramirez DW, Cano A (2011) Estado de la diversidad de la flora vascular de los Pantanos de Villa (Lima - Perú). Revista Peruana de Biología 17: 111-114.

Rovai AS, Coelho-JC, de Almeida R, Cunha-Lignonc M, Menghinic RP, Twilleya RR, Cintrón-Molero G, SchaefferNovelli $Y$ (2021) Ecosystem-level carbon stocks and sequestration rates in mangroves in the CananéiaIguape lagoon estuarine system, southeastern Brazil. Forest Ecology and Management 479: 118553. DOI: 10.1016/j.foreco.2020.118553

San Martín C, Pérez Y, Montenegro D, Álvarez M (2011) Diversidad, hábito y hábitat de Macrófitos acuáticos en la Patagonia occidental (Región de Aisén, Chile). Anales del Instituto de la Patagonia 39: 23-41.

Tobías-Baeza A, Salvador-Morales P, Sánchez-Hernández R, Ruiz-Acosta SC, Arrieta-Rivera A, Andrade-Prado H (2019) Composición florística y carbono en la vegetación arbórea de un área periurbana en Tabasco, México. Ecosistemas y Recursos Agropecuarios 6: 369-376.

URS- CHE (2010) Informe final Asistencia técnica para el control de macrófitos: Mejora de la gestión de los embalses del Bajo Ebro. URS-CHE. Ministerio del Medio ambiente y medio rural y marino. Barcelona, España. 151p. 